\title{
Abuse of Alabukun consumption: A biochemical and histomorphological study in
}

\section{Rattus norvegicus rats}

\author{
Emmanuel Tonbra Egoro *, Ikhide Godwin Ilegbedion and Prudence Nkemdinim Okara \\ Department of Medical Laboratory Science, Faculty of Basic Medical Sciences, Niger Delta University, P.M.B. 071, \\ Wilberforce Island, Bayelsa State, Nigeria.
}

World Journal of Biology Pharmacy and Health Sciences, 2021, 08(01), 037-042

Publication history: Received on 04 August 2021; revised on 06 October 2021; accepted on 08 October 2021

Article DOI: https://doi.org/10.30574/wjbphs.2021.8.1.0092

\begin{abstract}
This study was aimed on the biochemical and histomorphological study of abuse in the consumption of alabukun powder in Rattus norvegicus rats. Five milliliter of blood specimen was collected into lithium heparin bottles from seven rats weighing $240 \pm 2 \mathrm{~g}$ respectively with each of them administered with $0.2 \mathrm{mg} / \mathrm{kg}$ alabukun powder daily for a period of two weeks (experimental group) while another seven rats weighing $240 \pm 2 \mathrm{~g}$ each were not administered with alabukun powder (control group). Thereafter alanine aminotransferase, aspartate aminotransferase, urea, creatinine and C-reactive protein were measured quantitatively in both groups of rats. The mean values of all the measured biochemical parameters in Rattus norvegicus rats in the experimental group were statistically significant $(\mathrm{p}<0.05)$ as compared to that in the control group. This established biochemical finding was in conformity with the histomorphological examination of the kidney and liver organs. In conclusion, this study has established that administration of $0.2 \mathrm{mg} / \mathrm{kg}$ of alabukun powder on daily basis for a period of two weeks on Rattus norvegicus rats may induce hepato-renal and inflammatory disorders. It is thus recommended that consumption of alabukun powder by humans should strictly be in compliance with its prescription. However, kidney, liver and inflammatory biomarkers should be quantitatively measured in humans that have abused its consumption with a view to ascertain their health status and prevent any deleterious risks.
\end{abstract}

Keywords: Alabunkun; Abuse; Biochemical changes; Histomorphological changes; Kidney; Liver; Rattus norvegicus rats

\section{Introduction}

Alabukun is a local drug that is used to treat pains, feverishness, headache and cold. It contains $760 \mathrm{mg}$ and $60 \mathrm{mg}$ of acetylsalicylic acid and caffeine respectively. The acetylsalicylic acid which is otherwise known as aspirin is absorbed rapidly from the stomach as well as small intestine via passive diffusion across the gastrointestinal tract and subsequently hydrolysed rapidly thus forming salicylic acid with the aid of esterases in the plasma and gastrointestinal tract. This acid is distributed widely in the body with the highest concentration found in liver, renal cortex, plasma, lungs and heart. In the liver it forms salicyluric acid and other metabolites after its metabolism by phase ii conjugation reaction [1].

It helps to reduce pain by preventing the production of platelet aggregation with the inhibition of adenosine in the body. If not used in line with its prescription, this drug may lead to side effects such as asthma, vertigo, nausea, swelling due to fluid accumulation, gastrointestinal tract erosion which may result in occult bleeding and reduced iron uptake with the subsequent deficiency of iron [2]. The use of aspirin indiscriminately has also been observed to be associated with

${ }^{*}$ Corresponding author: Emmanuel Tonbra Egoro

Department of Medical Laboratory Science, Faculty of Basic Medical Sciences, Niger Delta University, P.M.B. 071, Wilberforce Island, Bayelsa State, Nigeria.

Copyright $\Subset 2021$ Author(s) retain the copyright of this article. This article is published under the terms of the Creative Commons Attribution Liscense 4.0 
blood disorders like anaemia and cytopenia [3]. Its low doses have been reported to significantly reduce circulatory erythrocytes and leukocyte count which may be suggestive of inhibitory action of bone marrow homeopoiesis while its high dose may cause death of the blood vessels cells [4]. Caffeine on the other hand has numerous responses that are associated with the pharmacology and cellular responses such as stimulation of the central nervous system, cardiac muscle, increased urinary output and relaxation of the smooth muscle in biological systems [5].

This local drug which is relatively cheap is widely used by many Nigerians without the prescription of a physician. This indiscriminate use initiated the need to carry out this research work which is aimed on abuse of alabukun consumption: a biochemical and histomorphological study in Rattus norvegicusrats.

\section{Material and methods}

\subsection{Study area}

This study was carried out in Department of Medical Laboratory Science, Faculty of Basic Medical Sciences, College of Health Sciences, Niger Delta University, Wilberforce Island, Bayelsa State, Nigeria.

\subsection{Ethical clearance}

This study which got the ethical approval from the ethical committee of Niger Delta University, Wilberforce Island, Bayelsa State, Nigeria was carried out strictly in compliance with the National Guideline for animal usage in research.

\subsection{Scope of experimental design}

A packet of alabukun powder was bought in a Pharmacy store located in Yenagoa and stored following strictly the storage instruction as recommended by the manufacturer. This powder was used for this research work.

\subsection{Pilot study}

A pilot study was carried out in order to ascertain the minimum dose of alabukun powder that can cause $100 \%$ death $\left(\mathrm{LD}_{100}\right)$ in the experimental rats. A total of 4 (four) rats having approximately the same weight $(240 \pm 2 \mathrm{~g})$ were used and the $\left(\mathrm{LD}_{100}\right)$ was obtained with the administration of $2 \mathrm{mg} / \mathrm{kg}$ of alabukun powder.

Also a pilot study was carried out in order to ascertain the minimum dose of alabukun powder that can cause $50 \%$ death $\left(L_{50}\right)$ in the experimental rats. A total of 4 (four) rats having approximately the same weight ( $\left.240 \pm 2 \mathrm{~g}\right)$ were used and the (LD50) was obtained with the administration of $/ \mathrm{kg}$ of alabukun powder.

\subsection{Sub-chronic toxicity study}

In this study 7 (seven) Rattus norvegicus rats weighing $240 \pm 2 \mathrm{~g}$ each were administered orally with $0.2 \mathrm{mg} / \mathrm{kg}$ of alabukun powder for two weeks (experimental group) while another 7 (seven) Rattus norvegicus rats each which served as control group were not administered with alabukun powder.

At the end of this experiment, the control and experimental rats were anaesthetized using inhaled chloroform technique after which $5 \mathrm{ml}$ of blood specimen was withdrawn from the cardiac of each rat for biochemical investigations. The rats were subsequently sacrificed with the liver and kidneys excised, blotted dry and stored in $10 \%$ formalin for histomorphological study.

\subsection{Laboratory Procedures}

All reagents were commercially purchased and the manufacturers' standard operational procedure (SOP) were strictly adhered to.

\subsection{Measurement of alanine aminotransferase}

This was measured in accordance with the colorimetric method as described by [6] using reagents manufactured by Randox Laboratories Limited, 55, Diamond Road, Crumlin County, Antrim, BT294QY United Kingdom.

\subsection{Measurement of aspartate aminotransferase}

This was measured in accordance with the colorimetric method as described by [7] using reagents manufactured by Randox Laboratories Limited, 55, Diamond Road, Crumlin County, Antrim, BT294QY United Kingdom. 


\subsection{Measurement of urea}

This was measured in accordance with the Urease-Berthelot's method as described by [6] using reagents manufactured by Randox Laboratories Limited, 55, Diamond Road, Crumlin County, Antrim, BT294QY United Kingdom.

\subsection{Measurement of creatinine}

This was measured in accordance with the Jaffe reaction as described by [8] using reagents manufactured by Randox Laboratories Limited, 55, Diamond Road, Crumlin County, Antrim, BT294QY United Kingdom.

\subsection{Measurement of C-reactive protein}

This was measured in accordance with the Latex turbidimetry method as described by [6] using Spin react Diagnostic kit manual, Spain.

\subsection{Statistical analysis}

The results of this study were expressed as mean and standard deviation, while the differences between the rats in the control and experimental groups were assessed using the students' $t$ ' test. The results were considered statistically significant at $\mathrm{p}<0.05$.

\section{Results and discussion}

Table 1 presented data on the effect of chronic administration of alabukun on some renal function test parameters. Values were presented as mean \pm standard deviation of mean. There was a statistically significant elevation in the mean concentration of urea between the control $(3.3 \pm 1.4)$ and test $(12.0 \pm 4.9)$ at $\mathrm{T}$-value of 5.0, $<<0.05$ and a negative correlation.

Also the creatinine level was significantly elevated statistically in the test group $(77.0 \pm 2.6)$ compared with the control $(67.0 \pm 7.8)$ with a student $T$-value of 3.2 at $\mathrm{p}<0.05$.

Previous reports documented that elevation of plasma urea concentration could be as a result of renal disease or failure [9]. There was elevation in the concentration of urea in the test group as compared with that of the control group. Furthermore creatinine, the most endogenous marker in diagnosis and treatment of kidney disease was elevated significantly in the test group as compared with that of the control group.

Table 1 Effect of chronic administration of alabukun on some renal function parameters of Rattus norvegicus rats (experimental group) as compared with the Rattus norvegicus rats that were not administered with alabukun (control group)

\begin{tabular}{|l|c|l|c|l|l|}
\hline Parameters & Control & Test & T-value & P-value & R \\
\hline Urea $(\mathrm{mmol} / \mathrm{L})$ & $3.3 \pm 1.4$ & $12.0 \pm 4.9$ & 5.0 & $0.0007^{* * *}$ & 0.02 \\
\hline Creatinine $(\mu \mathrm{mol} / \mathrm{L})$ & $67.0 \pm 7.8$ & $77.0 \pm 2.6$ & 3.2 & $0.0107^{* *}$ & 0.02 \\
\hline
\end{tabular}
KEYS: Values are expressed as mean \pm standard deviation of man at p<0.05; R=effective pairing correlation; T=paired student test; ${ }^{*}=$ level of
significance

This biochemical finding as established in this present study is in conformity with the histomorphological examination of the kidney organ of the experimental rats which revealed toxicity as shown in figure 1. 


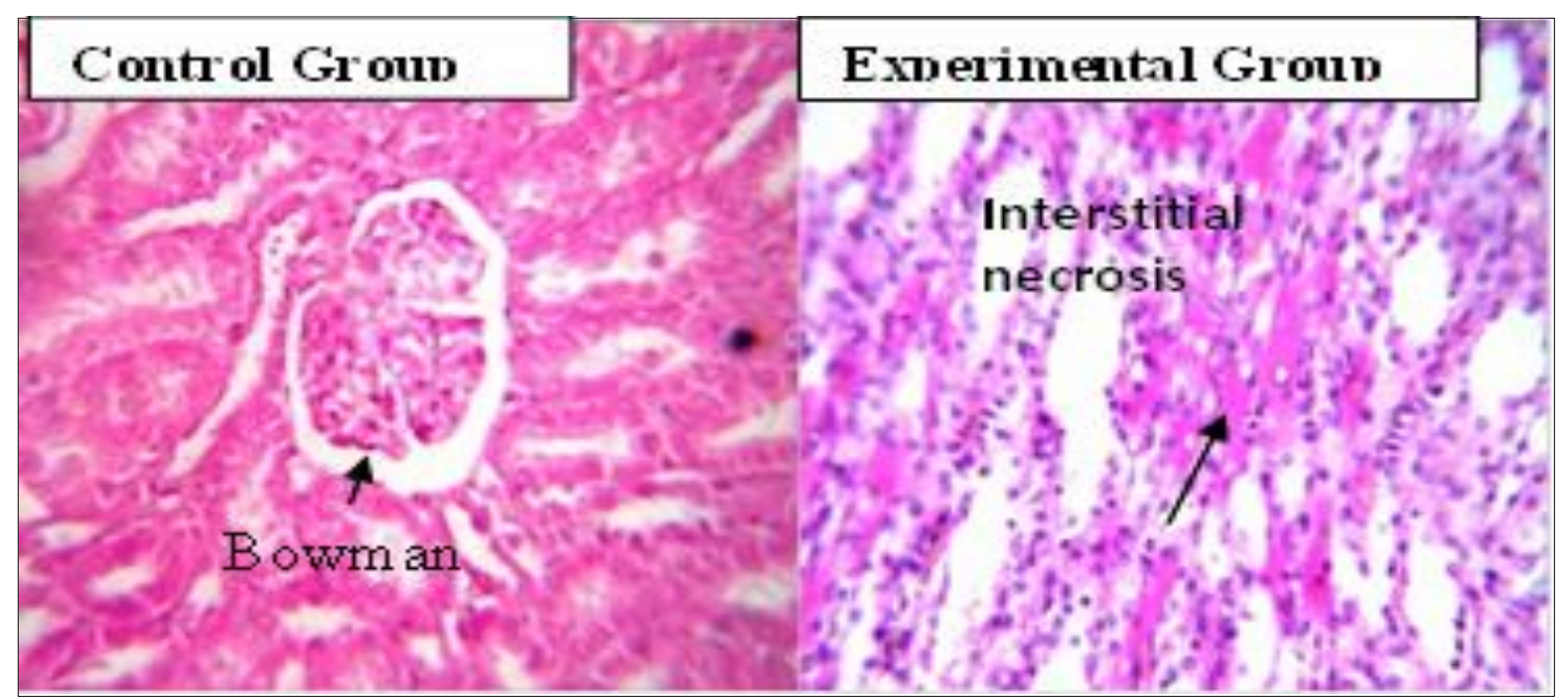

Figure 1 Photomicrograph of kidney slide showing toxicity as indicated by the arrow H\&E x400

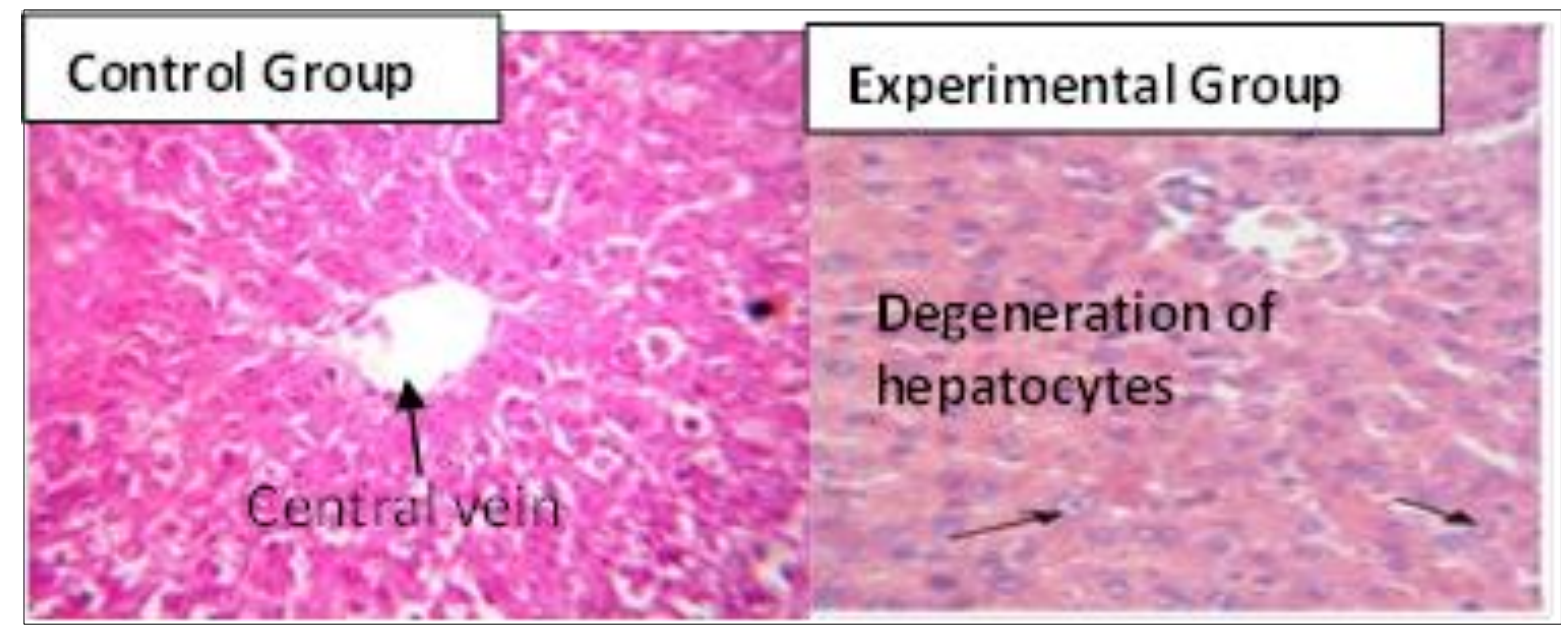

Figure 2 Photomicrograph of liver slide showing toxicity as indicated by the arrow H\&E x400

Table 2 Effect of chronic administration of alabukun on liver function test parameters and C-reactive protein of Rattus norvegicus rats (experimental group) as compared with the Rattus norvegicus rats that were not administered with alabukun (control group)

\begin{tabular}{|l|c|l|c|c|l|}
\hline Parameters & Control & Test & T-value & P-value & R \\
\hline AST (U/I) & $3.7 \pm 1.3$ & $16.0 \pm 5.5$ & 6.2 & $0.0002^{* * *}$ & 0.02 \\
\hline AST (U/I) & $4.4 \pm 1.6$ & $17.0 \pm 6.5$ & 6.7 & $0.001^{* *}$ & 0.08 \\
\hline CRP (mg/L) & $1.3 \pm 0.92$ & $4.4 \pm 6.5$ & 6.7 & $0.00 I^{* *}$ & 0.02 \\
\hline
\end{tabular}

KEYS: Values are expressed as mean \pm standard deviation of mean at $\mathrm{p}<0.05$; $\mathrm{R}=$ effective pairing correlation; $\mathrm{T}=$ paired student test; ${ }^{*}=$ level of significance; AST=aspartate aminotransferase; ALT=alanine aminotransferase

Table 2 shows data on the effect of chronic administration of alabukun on liver function test parameters and C-reactive protein of adult Rattus norvegicus rats. There was statistically significant elevation in the mean value of aspartate aminotransferase (AST) in the test group compared with the control (16.0 \pm 5.5 versus $3.7 \pm 1.3)$, T-value of 6.2 at $\mathrm{p}<0.05$. Also alanine aminotransferase (ALT) showed elevated values in the test group (17.0 \pm 6.5$)$ compared with the control $(4.4 \pm 1.6)$, T-value 6.7 at $\mathrm{p}<0.05$. The elevation of these two liver enzyme biomarkers in this study is symbolic 
of hepatocytes disorder. This finding is in line with [10] who reported that in toxin induced liver necrosis, hepatic ischemia and viral hepatitis there is increase in both enzymes.

Furthermore, C-reactive protein also exhibited a statistically significant elevation in mean value of the test group compared with the control group at $\mathrm{p}<0.05$ as shown in Table-2. All the three parameters showed negative correlation in terms of effective pairing. C-reactive protein is a protein synthesized by the liver in response to inflammation [11]. In this present study chronic administration of alabukun caused rapid elevation of C-reactive protein in the test group compared with the control group as established.

This biochemical finding as established in this present study is in conformity with the histomorphological examination of the liver organ of the experimental rats which revealed toxicity as shown in figure 2 .

\section{Conclusion}

In conclusion, this research work has shown that abuse of alabukun consumption may cause hepato-renal and inflammatory disorders.

\section{Recommendations}

Based on the findings from this research work it is recommended that:

- The abuse of alabukun powder consumption should be discouraged as this may be detrimental to human health.

- Consumers should comply strictly with its recommended prescription

\section{Compliance with ethical standards}

\section{Acknowledgments}

We acknowledge with thanks all those who assisted us in one way or the other during the course of this study.

\section{Disclosure of conflict of interest}

The authors declare no conflict of interest.

\section{Statement of ethical approval}

All the procedures used by the authors were in strict compliance with the ethical standards of National Guidelines for animal usage in research. Emmanuel Tonbra Egoro initiated this research work and was responsible for its title, aim, experimental design, analyses of the biochemical parameters, design of the tables and statistical analysis of the biochemical raw data while Ikhide Godwin Ilegbedion and Prudence Nkemdinim Okara were responsible for the sacrifice, excise and histomorphological examination of the liver and kidney organs of the rats as well as the interpretation of the findings. All the authors were responsible for the results and discussion as well as editing the references. Finally all the Authors proof read the work before it was submitted for publication.

\section{References}

[1] Marcia LB. Use of aspirin in children with cardiac disease, Pediatric Pharmacotherapy. 2007; 132: 4-12.

[2] Langman JS, Weil J, Wainright P, Lawson DH, Rawlings MD, Logan RFA.Risk of bleeding peptic ulcer associated with individual non-steroidal anti-Inflammatory drugs. Lancet. 1994; 334: 1075-1078.

[3] Dikshit P, Cha Herjee M, Goswami A, Mashra A, Jana NR. Aspirin induces apoptosis through the inhibition of proteasome function. J. Biol. Chem. 2006; 281(39): 29228-29235.

[4] Sandler RS, Halasi S, Baron JA, Buclinger S, Paskett E, Kereszies R. A randomized trial of aspirin to prevent colorectal cancer. N. Engl. Med. 2003; 348(10): 883-90.

[5] Dews BB. Stimulation of central nervous system and cardiac muscle by drugs. Ann. Rev. Nutr. 1982; 2: $323-341$. 
[6] Egoro ET, Oni ES, Chukwuma SA. Studies on selected biochemical and hormonal profile status in plasma of some roadside welders in Ajegunle, Nigeria. Global Journal of Medical Research K, Interdisciplinary. 2019; 19(6): 1723.

[7] Ifenkwe JC, Nwanjo HU, Nwosu DC, Solomon EA, Bot YS. Heavy metals cbioaccumulation and lipid peroxidation damage in residents of gas flaring community. International Journal of Advances in Scientific Research and Engineering. 2018; 4(7): 140-151.

[8] Obodo BN, Iyevhobu KO, Omolumen LE, Oaikhena FR, Alleh OA, Asibor E, Ifada SE. Renal function status in the elderly. World Journal of Pharmaceutical Research. 2000; 9(5): 75-84.

[9] Traynor J, Mactier R, Gaddes CC, Fox JG. How to measure renal function in clinical practice. British Medical Journal. 2006; 333: 733-737.

[10] Dufour DR, Lott HA, Noite Amr AA, Laa AH. Oxidative Stress Mediaters Drug-Induced Hepatotoxicity in Ratssa Possible Role of DNA Fragmentation Toxicology. 2005; 208: 367-375.

[11] Pepys MB, Hirschfield GM. C-reactive protein: A critical update. The Journal of Clinical Investigations. 2003; 111(12): 1805-1812. 